\title{
Lupus Eritematoso Diseminado en la Infancia Estudio Clínico y de Sobrevida en 31 Casos
}

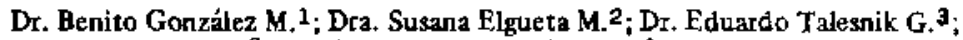 \\ Dr. Nelson Montaña N. ${ }^{3}$; Dra. M. Soledad Rodríguez T.4; Dra. Marta Valenzuela D.5; \\ Dra. Marta Miranda $\mathbf{A}^{5}$; Dra. Mónica Varas P.6 \\ Systemic Lupus Erythematosus in Childhood \\ Climical Study and Survival in 31 Patients
}

\begin{abstract}
Thirty one patients, aged 4 to 15 years old with Systemic Lupus Erythematosus (SL.E) were evaluated jetrospectively in order to analyse the clinical course of the disease. Twenty six patients were female and 5 males. All of them fulfiled the criterions stated by the American Rheunatologist Academy for the diagnosis of S.L.E. Clinical and laboratory diagnostic parameters were similar to those of adult type. Our patients had mainly articular and cutancous lessions. Whole survival rates were 68 and 40 per cent at five and ten years respectively. In children with renal lupus lesions the survival rates disminished to 57 and 28 per cent. Patients without renal involvement had 90 per cent survival rates along the whole 10 years period. The authors rise the possibility to lower mortality rates, in those patients presenting initially with renal S.I.E. damage, by adding to their treatrnent other inmunosuppressors to the usually prescribed adrenal steroid derivates, because anong 19 patients with such an evidence, only $5 / 12$ (42\%) receiving steroids alone survived at 5 years follow up in contrast with $7 / 7$ of those receiving combined schedules.
\end{abstract}

La sobrevida del paciente con Lupus Eritematoso Diseminado (LED) infantil ha sido materia de controversia debido a los diferentes resultados que han publicado en los últimos años. En efecto, los primeros trabajos. demostraban sobrevidas a 5 años de $72 \%$ y $42 \%$ en niffos sin y con compromiso renal respectivamente, en cambio publicaciones más recientes đan sobrevidas de $100 \%$ en pacientes con lesión renal ${ }^{2}$.

Estos resultados pueden explicarse, en gran medida, por los avances alcanzados en el diagnóstico mediante la introducción de nuevas técnicas inmunológicas que permiten hacerlo más precozmente, un mejor manejo de la actividad lúpica y por otra parte, el uso de corticosteroides y antibióticos que ha significado controlar en mejor forma la enfermedad y evitar las complicaciones infecciosas que constituyen una de las principales causas de mortalidad en estos pacientes ${ }^{3}$.

No obstante estos adelantos, queda aún por resolver el papel que tienen los nuevos tratamien. tos, especialmente las drogas inmunosupresoras

I Jefe Unidad Inmunalogía - Reumatología. Hospital Luis Calvo Mackena, Santiago.

2 Facultad de Medicina. Universidad de Chile, Santiago.

${ }^{3}$ Servicio de Pediatría. Hospital Josefina Martínez, Santiago.

4 Servicio de Pediatría, Hospital Guillermo Grant Benavente, Concepción.

S Servicio de Reumatología, Hospital San Juan de Dios, Santiago.

6 Servicio de Pediatría, Hospital Roberto del Ría, Santíago. en el pronóstico de esta enfermedad. Algunos autores ${ }^{4}$ sostienen que el pronóstico de LED infantil ha mejorado considerablemente con el uso de una terapia más agresiva, combinando esquemas de corticosteroides y agentes inmunosupresores. Otros ${ }^{2}$ piensan que el uso de fárma$\cos$, distintos a los esteroides, no tendria influencias positivas sobre el pronóstico de estos pacientes.

No existiendo en nuestro país, un estudio clínico y pronóstico de un número suficiente de pacientes con LED infantil, queremos dar a conocer los resultados de un análisis retrospectivo de las principales manifestaciones clínicas y sobrevida de nithos afectados realizado en forma cooperativa con la participación de cinco centros pediátricos nacionales.

\section{PACIENTES Y METODOS}

Para el intervalo comprendido entre los años 1969 y 1980, se pudieron analizar 33 enfermos en los cinco centros que participaron en este estudio: Hospital Luis Calvo Mackenna (17 casos), Hospital Josefina Martínez (4 casos), Hospital San Juan de Dios (3 casos), Hospital Roberto del Río (4 casos) y Hospital Guillermo Grant Benavente (5 casas). De los 33 pacientes, se han incluido en la presente investigación solamente 31 , todos menores de 16 años, que reunían por lo menos 4 de los criterios propuestos por la Academia Reumatológica Americana (ARA) para el diagnóstico de $L^{2} D^{5}$. En los 2 
pacientes excluidos se comprobó Lupus Eritema. toso inducido por drogas anticonvulsivas.

Durante este periodo de observación, los pacientes fueron sometidos, en general, a pautas semejantes de estudio y evolución clínica.

El tratamiento realizado consistio en Predniso. na $2 \mathrm{mg} \mathrm{x} \mathrm{Kg/diario} \mathrm{en} 19$ casos; Prednisona más Acido Acetil Salicílico (ASA) en 3 casos; Prednisona con Cloroquina en 4 casos; Prednisona con ASA y Azathioprina en 2 casos; Prednisona $y$ Azathioprina en 1 caso y Prednisona con Ciclofosfamida en dos casos.

Se estableció que existía compromiso renal ante el hallazgo de cualquiera de los siguientes elementos:

Proteinuria de 24 horas superior a 1 g. o/oo; Hematuria con recuento de Addis con un número de hematíes superiores a 5 células por campo; Cilindruria; Creatininemia 150 umol/1; confirmación de daño en estudio microscópico de biopsias renales.

Solamente en 15 pacientes se pudo realizar estudio histopatológico renal $(48,3 \%)$, clasificándose las nefropatías según los criterios propuestos por Baldwin y col. ${ }^{6}$.

En el análisis de sobrevida de nuestros pacientes, se aplicó el método de Cutter y Ederer ${ }^{7}$ tomando como fecha inicial el momento en que fue confirmado el diagnóstico de LED infantil.

\section{RESULTADOS}

La edad del diagnóstico y el sexo de los 31 pacientes se detallan en la Figura 1 en donde 26 pacientes eran del sexo femenino $(83,8 \%)$ y 5 de sexo masculino (16,2\%). La edad de diagnóstico fluctuó entre los 4 y 15 años, destacándose una incidencia mayor entre los 10 y 15 afios de vida (22 casos).

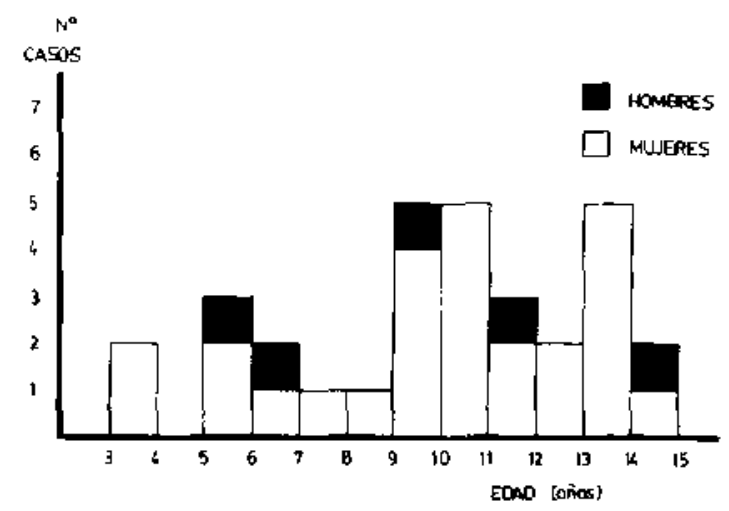

Figura 1. Edad de comienzo y sexo en 31 pacientes con LED Infantil.
Las principales características clínicas en las cuales se orientó el diagnóstico de enfermedad lúpica se muestran en la Tabla 1.

Tabla 1.

Manifestaciones clínicas iniciales en 31 LED infantil

Manifestaciones

Compromiso del estado general

Poliartralgias - Poliartritis

Fiebre

Lesiones cutáneas

Visceromegalia - Poliadenopatía

Compromiso pleural

Compromiso renal

Fenómeno Raynaud

Compromiso neurológico

Anemia hemolítica

Púrpura Trombocitopénico

$\begin{array}{cr}\text { No Casos } & \% \\ 25 & 80 \\ 25 & 80 \\ 21 & 67 \\ 19 & 61 \\ 12 & 39 \\ 7 & 22 \\ 5 & 16 \\ 4 & 13 \\ 3 & 10 \\ 2 & 6 \\ 1 & 6\end{array}$

Como se puede apreciar, existe un claro predominio del compromiso del estado general, articular y cutáneo en la mayoría de nuestros pacientes. Hubo dos enfermos que ingresaron con el diagnóstico de Síndrome febril prolongado, cuya etiología fue aclarada después de 35 y 60 días respectivamente. En los otros, la fiebre constituyó una de las manifestaciones iniciales de la enfernedad.

El compromiso articular se caracterizó por artralgias de una o más articulaciones en 23 enfermos (74\%) y solamente en dos se evidenciaron signos de artritis franca, en uno de rodilla $y$ en otro de tobillo.

De 19 ninos que mostraban eritema facial con đistribución tipica (mariposa lúpica), en dos enfermos, al cabo de seis meses de evolución, las lesiones cutáneas eran tan extensas que comprometian gran parte del tórax y el dorso, se acompañaban de zonas de vasculitis y disminuyeron al aumentar la dosis de tratamiento esteroides.

El compromiso pulmonar con que comenzó la enfermedad en 7 niffos, se caracterizó en todos por derrarne pleural de mediana intensidad, seguido posteriormente por las manifestaciones articulares. Hay que hacer notar que en dos enfermos, junto con el compromiso pleural, se pudo observar en diferentes estudios radiológicos practicados, zonas de condensación pulmonar retrocardiaca izquierda, sugiriendo el diagnóstico de TBC pulmonar, que se desechó solamente por el fracaso terapéutico y la evolución clínica.

Los síntomas neurologicos ocurrieron en un riño como síndrome convulsivo que hizo pensar en epilepsia, descartada al aparecer otras manifes- 
taciones sistémicas después de cuatro meses de observación; en otra enferma que injcio sus molestias con Corea y compromiso articular, $y$ en un paciente de 13 años que cornenzó con mielitis transversa, retención urinaria y posteriormente compromiso hematológico severo y púrpura, cuya aparición orientó finalmente al diagnóstico correcto.

En cinco enfermos el LED comenzó dando sintomas de compromiso renal, como síndrome nefrótico $(n=4)$ y hematuria $(n=1)$. En tres de ellos hubieron simultáneamente signos de insuficiencia renal, gran compromiso del estado general y alteraciones de otros parénquimas.

La anemia hemolitica no fue frecuente; solamente dos enfermos la presentaron, uno sufrió durante dos afios crisis intensa de hemólisis $y$ sólo después de una esplenectomía, tuvo otros sintomas que permitieron el diagnóstico de LED.

Otro síntoma relativamente frecuente en nuestra serie fue la alopecía, evidente en $40 \%$ de los enfermos.

Entre las alteraciones hematológicas más frecuentes estuvieron la VHS elevada y la anemia. La anemia fue normocítica, normocrómica en 27 enfermos; microcítica, hipocrómica en dos y en otros 2 hemolítica autoinmune con reacción de Coombs positiva (Tabla 2).

Tabla 2.

Laboratorio en LED infantil

\section{Exámenes}

Alteraciones Hematológicas:
VHS elevada
Anemia
Leucopenia
Trombocitopenia

Alteraciones Inmunologicas:

Células LE (t)

$A A N \geqslant 1 / 200$

Anti-DNA

Hipergamaglobulinemia

Complemento $\mathrm{C}_{3}$ bajo

Factor reumatoídeo

Déficit de lgA

Pacientes (\%)

96.77

64.51

32.25

9.67

90.32

80.00

66.00

48.00

48.00

23.00

3.00
La búsqueda de células de Lupus (LE) dio resultados positivos en 90\% de los casos y de anticuerpos antinucleares (AAN) en el 80\%: de estos últimos, $30 \%$ con títulos de $1 / 200$ y en el $70 \%$ restante iguales o superiores a $1 / 400$. Los resultados de los anticuerpos antj-DNA (A.DNA) fueron positivos en $66 \%$ de los casos y se correlacionaton bien con los AAN salvo en un caso en que estos habían sido negativos. De los
31 enfermos, solo uno tení deficit selectivo de IgA sérica $(<10 \mathrm{mg} \%$ ) y no mostró diferencias evolutivas con el resto de los pacientes que tenían concentraciones normales.

19 enfermos tenían, en el momento del diag. nóstico de LED, sedimentos urinarios alterados $(61,29 \%)$, caracterizados por proteinuria en 17 , cilindruria en 14 , hematuria en 10 y pruebas de función alteradas con depuración de creatinina < $90 \mathrm{~m} / \mathrm{min} / 1.73^{2}$ en 7 enfermos. En 15 pacientes con manifestaciones urinarizs, se realizó biopsia renal, cuyos resultados y correlación con otros parámetros clinicos y de laboratorio se detallan en la Tabla 3. Como se puede observar, la lesión histo-patológica más frecuente, según la clasificación de Baldwin, fue la Glomerulonefritis Proliferativa focal (G.N.P.F.) en 8 enfermos (53\%), Glomerulonefritis Proliferativa difusa (G.NP.D.) en $6(40 \%)$ y solamente 1 paciente con Glomerulonefritis Mesangial (7\%).

No siempre se encontró buena correlación entre los hallazgos urinarios y la lesión histológ. ca, pues hubo enfermos con intensas alteraciones urinarias cuya biopsia demostró G.N.P.F. (5 casos) y por otra parte, enfermos que presentaron pocos cambios en el examen de orina y el resultado histologico arrojó una G.N.P.D. (2 casos). En general, exjstía buena correlación entre niveles bajos de $\mathrm{C}_{3}$ y compromiso renal, salvo en 4 enfermos, todos portadores de G.N. Focal.

El perfodo de observación promedio en nuestros pacientes fue de $\mathbf{4 2 . 2 1}$ meses con un rango de 1 a 144 meses, si se toma como fecha inicial la del diagnóstico de LED.

La sobrevida global de estos nifios fue de 68 y $40 \%$ a los 5 y 10 afios de observación respectivamente (Fig. 2). Cuando se separaron los nifros con compromiso renal inicial se vió que 12 pacientes que ingresaron sin lesión renal, tuvieron una sobrevida a los 5 y 10 años plazo de $90 \%$. El períoda promedio de observación de ellos alcanzó a 42,41 meses en un rango que fluctuó entre 12 - 144 meses. Para el grupo con nefropatía (19 casos), la sobrevida a 5 y 10 años alcanzó solamente a 57 y $28 \%$ respectivamente. Este último grupo, tuvo un período promedio de observación semejante al anterior, de 42,68 me. ses con rango de 1 a 114 meses. La diferente sobrevida de ambos grupos es estadísticamente significativa $(P<0,05)$.

Con el fin de estudiar algunas de las posibles influencias sobre la elevada mortalidad de estos niños, se comparó el pronóstico con el tipo de tratamiento recibido, el que consistió tal como se citara anteriormente, en un grupo de 19 nifios manejados exclusivamente con Prednisona $y$ otros 12 pacientes que recibieron tratamiento combinado (Prednisona + Cloroquina $=4$ casos; 
Tabla 3.

Compromiso Renal en LED Infantil. Correlación Anátoma - Clínica

\begin{tabular}{|c|c|c|c|c|c|c|}
\hline \multirow[b]{2}{*}{ Paciente } & \multirow[b]{2}{*}{ Forma Clínica } & \multicolumn{2}{|c|}{ Sedimen to Urinario } & \multirow[b]{2}{*}{ Hematies } & \multirow[b]{2}{*}{$\mathrm{C}_{3}$} & \multirow[b]{2}{*}{ Biopsia Renal } \\
\hline & & Protefnas & Cilindros & & & \\
\hline 1. S.T.R. & Articular & +1 & $(-)$ & $+1+$ & $\downarrow$ & G.N. Difusa \\
\hline 2. C.S.G. & Articular & $+1+$ & $+1+$ & + & $\downarrow$ & G.N. Mesangial \\
\hline 3. J.M.P. & Articular & $+1+$ & $+1+$ & $(+)$ & $\downarrow$ & G.N. Focal \\
\hline 4. M.C.F. & Articu & $(-)$ & $(-)$ & $(-)$ & N & G.N. Focal \\
\hline 5. P.M.T. & Articular & $(t)$ & $(-)$ & $(-)$ & $\downarrow$ & G.N. Difusa \\
\hline 6. M.A.G. & Asticular & $+H$ & +++ & $H$ & $\mathbf{N}$ & G.N. Difusa \\
\hline 7. S.G.C. & Articular & + & $H$ & + & $\downarrow$ & G.N. Difusa \\
\hline 8. R.R.C. & S. Nefrótico & $+1+$ & $(-)$ & $(-)$ & $\downarrow$ & G.N. Focal \\
\hline 9. M.O.S. & Corea & + & + & + & $t$ & G.N. Focal \\
\hline 10. R.R.H. & S. Nefrótico & $+1+$ & + & ++ & $N$ & G.N. Focal \\
\hline 11. I.M.C. & Articular & + & $(-)$ & $(-)$ & N & G.N. Focal \\
\hline 12. I.M.H. & S. Nefrática & $+H+$ & + & $+1+$ & N.R. & G.N. Difusa \\
\hline 13. R.M.R. & A. Hemolítica & ++ & ++ & $(-)$ & N & G.N. Focal \\
\hline 14. M.M.A. & Articular & + & ++ & $(-)$ & $\downarrow$ & G.N. Difusa \\
\hline 15. M.B.S. & P. Trombocitopénico & + & + & $+1+$ & $\downarrow$ & G.N. Focal \\
\hline
\end{tabular}

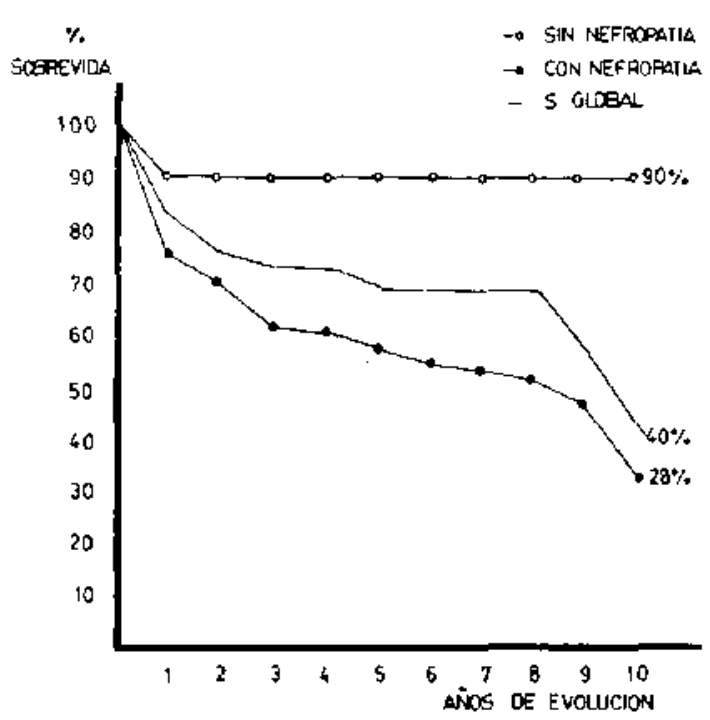

Figura 2. Sobrevida de 31 pacientes con LED Infantil.

Prednisona + Ciclofosfamida $=2$ casos; Prednisona + Azathioprina $=3$ casos; Prednisona + ASA $=3$ casos). Los resultados de ambos tipos de terapia observan en la Fig. 3 donde se puede apreciar que los 19 pacientes que sólo recibieron Prednisona tuvieron una sobrevida a 5 y 10 años de 48 y $19 \%$ respectivamente. Por su parte, en los 12 niños que recibieron terapia combinada, la sobrevida alcanzó a $91 \%$ a 5 y 10 años de observaciốn. El análisis estadístico de la frecuencia de lesión renal entre ambos grupos, no demos. tró diferencias importantes $(\mathrm{p}=>0,05)$.

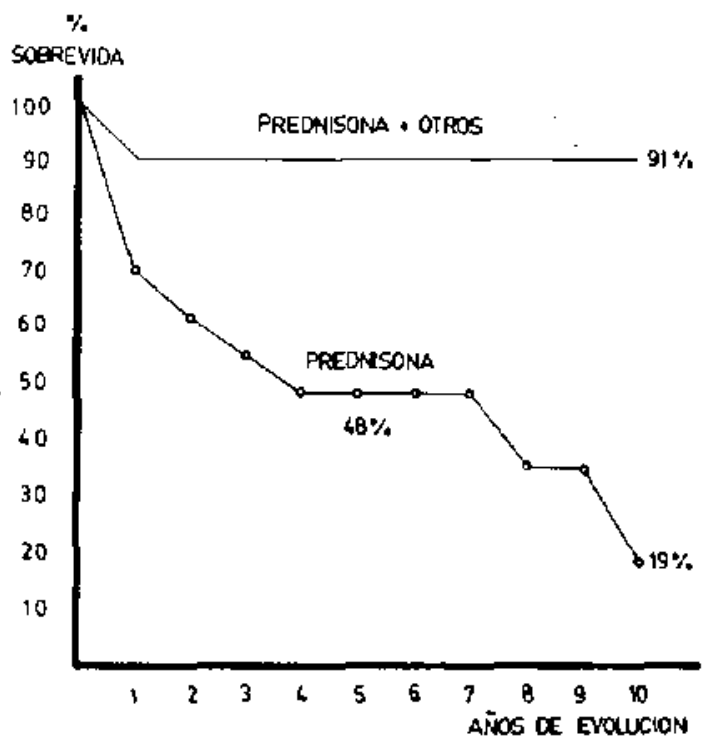

Figura 3. Sobrevida según tipo de tratamiento en LED Infantil.

Por otra parte, a los 5 años los 7 enfermos con nefropatía lúpica que recibieron tratamiento combinado estaban vivos, en cambio 5 de 12 tratados exclusivamente con prednisona habían 
fallecido. No hubo preponderancia de ningún tipo histológico en ambos grupos, distribuyéndo. se en forma equivalente.

De los 31 enfermos analizados, 12 fallecieron durante este período $(38,7 \%)$, con un promedio de sobrevida de 32,58 meses y un rango de 1 a 168 meses. La mitad de las muertes ocurrió antes de los dos años de tratamiento y las restantes, entre 2 y 9 anos. De los pacientes fallecidos $91 \%$ recibió tratamiento exclusivo con Prednisona y solamente el $8,3 \%$ recibió tratamiento combina. do.

Las causas de la muerte más frecuentes en estos nifios fueron insuficiencia renal en 6 pacientes $e$ infecciones bacterianas en 3 . En los 3 restantes no fue posible conocerla con precisión.

\section{DISCUSION}

El análisis de las manifestaciones clinicas injciales en nuestros enfermos, no mostró diferencias con las que clásicamente se describen para los pacientes adultos con LED, predominando el compromiso articular y cutáneo en más del $60 \%$ de estos nirros. Habria que hacer notar que el LED infantil, suele confundirse con otras afecciones. Las confusiones más frecuentes ocurrieron en casos que comenzaron con manifestaciones neurológicas, hematológicas o ambas, en ellos el diagnóstico se estableció definitivamente sólo después de algún tiempo de observación. La aparición de otros fenómenos autoinmunes puede orientar hacia la verdadera causa de los síntomas.

Entre los estudios de laboratorio realizados en estos pacientes, la presencia de AAN y ADNA en el 80 y $66 \%$ respectivamente, confirma el valor de ambos procedimientos en el diagnóstico $y$ evaluación de la enfermedad tal como se ha descrito en varias publicaciones nacionales y extranjeras ${ }^{8 \cdot 9}$. La combinación de ambas técnicaa, ha permitido aumentar su especificidad, pues la frecuencia con que se detectan los anticuerpos anti-DNA en otras mesenquimopatías es muy baja. Esto constituye un notable adelanto para la pediatría pues en muchos casos de LE, las manifestaciones clínicas son semejantes a las de Ia Artritis Reurnatoidea Juvenil. Debe tenerse en cuenta, sin embargo, que en la Enfermedad mixta del tejido conectivo $17 \%$ de los pacientes tienen títulos positivos a ADNA ${ }^{9}$.

La presencia de anticuerpos ADNA en 3 niffos sin compromiso renal aparente, viene a destacar que la positividad de este examen no siempre guarda relación con la evidencia de daño o actividad lúpjca renal.

El hallazgo de compromiso renal en $62 \%$ de los pacientes en esta enfermedad, es concordante con lo descrito por otros autores ${ }^{10}$ y apoya el concepto de que en el adulto $y$ en el niño, el rifión es frecuentemente comprometido por el deposito de complejos inmunes. Ha sido interesante comprobar que existe escasa correlación entre los resultados de los exámenes urinarios y la histopatología renal. En general, se podria afirmar que la G.N. Difusa y Membranosa son las que producen mayores alteraciones en el sedimento urinario con proteinurias masivas, hematuria o ambas. En cambio en la G.N. Focal es posible observar proteinurias de menor magnitud. Hay que hacer notar que algunas G.N. Focales diagnosticadas con microscopia corriente, corregponden más bien a G.N. Membranosas en el microscopio electrónico. Esto podria explicar la proteinuria importante encontrada en un paciente (RRD) con G.N. Focal en quién se realizó solamente microscopia óptica.

Las cifras de sobrevida favorecen notablemente a los pacientes sin đaño renal confirmando que el compromiso renal constituye un elemento pronóstico muy importante en esta enfermedad 10.

La sobrevida global de esta serie es inferion a la de otras publicaciones recientes, donde $\boldsymbol{*}$ aprecian cifras superiores a $80 \%$ en 5 y 10 años de observación: Abeles y col. ${ }^{2}$ dan cifras de $93 \%$ a cinco años; Caeiro y $\operatorname{col}^{11}$ de $82 \%$; Fish observó una sobreviđa global a 10 años de $86 \%$ (Tabla 4). En el análisis de estos trabajos, destaca que siempre se usó Prednisona con drogas inmunosupresoras cuando había evidencia de daño renal o dosis convencionales de corticosteroides no lograban controlar la enfermedad.

En nuestra serie los enfermos tratados solamente con Prednisona tuvieron un pronóstico muy malo en comparación con los tratados con esquemas mixtos, más agresivos. La diferencia es estadisticamente significativa y no podría explicarse por una mayor cantidad de pacientes con nefropatía, en el grupo tratado con Prednisona ( $12 / 19$ vs $7 / 12$ en el grupo manejado con esquemas combinados), porque el análisis estadístico no pernite detectar diferencias significativas en la frecuencia de nefropatia entre ambos grupos. La sobrevida de los niños portadores de compromiso renal sugiere que el tratamiento indicado tuyo gran influencia sobre la evolución posterior. Fue así como en los 12 casos tratados con Prednisona exclusivamente, la sobrevida a 5 años plazo, no superó al $42 \%$ mientras que en el grupo tratado con esquemas mixtos, todos los pacientes estaban vivos al término de dicho periodo de observación.

Creemos que la mayor mortalidad de nuestra serie respecto a otras publicaciones podria expli- 
Tabla 4.

Sobrevida del LED Infantil en la Literatura Extranjera

\begin{tabular}{|c|c|c|c|c|c|c|c|c|}
\hline \multirow[t]{2}{*}{ Autores } & \multirow{2}{*}{$\begin{array}{l}\text { Año } \\
\text { Publi- } \\
\text { cación }\end{array}$} & \multirow[t]{2}{*}{ Tratamiento } & \multicolumn{3}{|c|}{$\begin{array}{l}\text { Sobrevida a } \\
5 \text { Años }\end{array}$} & \multicolumn{3}{|c|}{$\begin{array}{c}\text { Sobrevida a } \\
10 \text { Años }\end{array}$} \\
\hline & & & Global & S/Nef. & C/Nef. & Global & S/Ner. & $\mathrm{C} / \mathrm{Ner}$. \\
\hline Meislin y Col. & 1968 & Esteroides & --- & $72 \%$ & $42.0 \%$ & 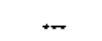 & - & - \\
\hline Mota y Col. & 1975 & & - & -- & $50.0 \%$ & - & - & $\cdots$ \\
\hline Garin y Col. & 1976 & Pred. + Inmunosupres. & - & - & $60.9 \%(+)$ & $\rightarrow$ & $\sim$ & - \\
\hline Fith y CoL & 1977 & Pred. + Azathioprina & - & -- & - & $86 \%$ & $72 \%$ & $73 \%$ \\
\hline Abeles y Col. & 1980 & Pred, + Cloroquina & $93 \%$ & $100 \%$ & $89.0 \%$ & -- & - & - \\
\hline Caciro y Col. & 1981 & Pred. + Azathioprina & $82 \%$ & $100 \%$ & $59.0 \%$ & $76 \%$ & $100 \%$ & $47 \%$ \\
\hline González y Col. & 1982 & Prednisona & $68 \%$ & $90 \%$ & $57.0 \%$ & $40 \%$ & $90 \%$ & $28 \%$ \\
\hline
\end{tabular}

(+) Pacientes con G.N.P. Difusa.

carse como consecuencia de los siguientes hechos:

Cuatro enfermos llegaron en una etapa terminal y fueron tratados solamente con corticoides; no se utilizaron inmunosupresores en un buen número de enfermos 'con nefropatía lúpica; no todos los pacientes fueron manejados con la ayuda de las técnicas inmunológicas más recientes de evaluación de esta enfermedad como AAN, ADNA, $\mathrm{C}_{3}$, y otras.

Estas observaciones sugieren la necesidad de usar esquemas de tratamiento más agresivos, que si bien para algunos autores son aún discutibles, parecen producir resultados más favorables que aquellos que utilizan exclusivåmente esteroides. Por otra parte, parece muy probable que con la incorporación de nuevas técnicas de análisis inmunológico se podrán observar resultados más satisfactorios en el futuro, como consecuencia de un manejo más preciso de cada situación.

\section{RESUMEN}

Se 'efectuó un análisis clínico y evolutivo de 31 pacientes portadores de Lupus Eritematoso Diseminado (LED) de manera retrospectiva. Todos los sujetos eran a menores de 16 años de edad y reunían como mínimo 4 de los requisitos exigidos por la Academia Reumatológica Americana (ARA) para el diagnóstico de la enfermedad.

De los 31 pacientes, 26 pertenecieron al sexo femenino y 5 al masculino. Las principales manifestaciones clínicas y de laboratorio fueron semejantes a las descritas para el LED de tipo adulto, predominando las lesiones articulares y cutáneas. Se subraya la contribución de las nuevas técnicas inmunológicas en el diagnóstico y evolución de la enfermedad, especialmente de los AAN y AADN.
La sobrevida global en nuestros enfermos alcanzó a 68 y $40 \%$ en 5 y 10 afios respectivamente, disminuyendo a 57 y $28 \%$ en los que sufrían nefropatía lúpica. Los enfermos sin lesión renal, tuvieron $90 \%$ de sobrevida durante el periodo de observación de 10 años.

Se analiza el impacto de los distintos tratamientos en la enfermedad y sugiere que el uso de combinaciones de esteroides suprarrenales con inmunosupresores, pudiera reducir la elevada mortalidad observada en estos pacientes.

\section{REFERENCIAS}

1 Meislin, A.G.; Rothfield, N.: Systemic lupus ery thematosus in childhood. Analysis of 42 cases, with comparative data on 200 adult cases followed concurrenty. Pediatrics 42: 37, 1968.

2 Abeles, M.; Urman, J.; Weistein, A.; Lowenstein, M.; Rothfield, $N$.: Systemic Lupus Ery thematosus in the younger patients: Survival studies. J. Rheumatol. $7: 512,1980$.

${ }^{3}$ Urman, J,; Rothfield, $N_{\text {.: }}$ Corticostezoid treatment in Systemic Lupus Erythematosus. Survival Studies. J.A.M.A. 238: 2.272, 1977.

4 Fish, A,; Blau, E.; Westberg, N., Burke, B.; Vemier. R.; Michael, A.: Systemic Lupus Erythematosus within the first two decades of life. The J. Med. 62: 99,1977 .

${ }^{5}$ Cohen, A.S.; Reynolds, W.E.; Franklin, E.C. et al.: Preliminary criteria for the classification of Systemic Lupus Erythematosus. Bull Rheum. Dis. 21: 643, 1971.

${ }^{6}$ Baldwin, D.S.; Lowestein, J.; Rothfield, N.F. et al.: The clinical course of the proliferative and membranous corms of lupus nephritis. Ann. Intern. Med. 73 : $929,1970$.

7 Cutler, S.J.; Ederer, F.: Maximun utilization of the life table in analysing survival. J. Chronic. Dis. 8: $699,1958$.

- Guzmán, L.; Gonzấlez, C.; Arriaguda, M.: Anticuerpos antinucleares y anti-DNA nativo en mesenquimopatías. Rev. Med. Chile 1 10: 24, 1982. 
9 Lehman, T.; Singsen, B; Bernstein, B.: The role of antibodies directed against double-stranded DNA in the manifestations of systemic jupus ery thematosus in Chitdhood. J. Pediatr. 96:657, 1980.

10 Garin, E.; Donnelly, W.; Fennell, R.; Richard, G.: Nephiritis in systemic lupus ery thematosus in children. J. Pediatr. 89: 366, 1976.

11 Caerio, $F_{\text {.; }}$ Michielson, R,; Bernstein G.; Hughes, V; Ansell, B.: Systemic lupus ery thematosus in child. hood. Ann. Rheum. Dis 40: 325, $19 B 1$.

12 Sigsen, B.; Bermstein, B.; Koster, K, K, Hanson, V.:
- Systemic lupus ery thematosus in childhood: Correlations between changes in disease activity and senum complement levels. J. Pediatr. 89: 358, 1976.

${ }^{13}$ Miniter, $M$.; Stollar, D.; Agnello, $V .:$ Reassessment of the clinical significance of native DNA intibodies in systemic lupus erythematosus. Arthritis Rheum. 15: $174,1972$.

${ }^{14}$ Mota, F.: Velózquez, L.; Zuniga, V.; Gordillo, S.: Evolución de la nefropatía lúpica en niños. Bol. Med. Hosp. Infant Mex. 32: 787, 1975. 